\title{
PERCEPCIÓN DEL CAMBIO INTITUCIONAL EN LA UNIVERSIDAD PÚBLICA
}

\author{
Jorge Ernesto Quintero Félix \\ Universidad Autónoma de Sinaloa \\ Correo e: jeqf@uas.uasnet.mx \\ Víctor Antonio Corrales Burgueño \\ Universidad Autónoma de Sinaloa \\ Correo e: tonni@uas.uasnet.mx \\ Gloria Aréchiga Sánchez \\ Universidad Autónoma de Sinaloa \\ Correo e: garechi@uas.uasnet.mx \\ Marcela Rebeca Contreras Loera \\ Universidad de Occidente \\ Correo e: marcelac25@hotmail.com
}

\section{RESUMEN:}

El presente trabajo aborda la percepción de los académicos sobre el cambio que experimentan sus instituciones. El problema se abordó tomando como caso a la Universidad Autónoma de Sinaloa. Su propósito es conocer cómo el sector académico valora o procesa el impulso de modernización de estos centros de estudio; para ello, es importante conocer la proclividad manifiesta de los académicos para ser partícipes de dicho proceso, así como los efectos que trae consigo en términos de convergencia o pérdida de cohesión e identidad institucional. Para dar soporte interpretativo al fenómeno en cuestión, se muestran de inicio las distintas concepciones que actualmente se debaten sobre las teorías del cambio social y de las organizaciones, tomando postura por aquélla que resalta la importancia que (en condiciones de inestabilidad y turbulencia) adquiere el contexto social para las organizaciones universitarias.

Palabras clave: Organización. Universidad. Cambio.

\section{PERCEPTION OF INSTITUTIONAL CHANGE IN THE PUBLIC UNIVERSITY}

\begin{abstract}
:
This paper tackles the perception by academics regarding the changes their institutions are facing. The problem is studied by looking at the Autonomous University of Sinaloa. The purpose is to know how the academic sector values or processes the momentum of modernization in their study centers; therefore it is important to understand the apparent inclination of the scholars to be part of the modernization process and the effect that it brings in terms of convergence or loss of cohesion and institutional identity. To support the interpretation of the phenomenon in question, the different conceptions, that nowadays are in debate about the theories of social change and organizations are shown, enhancing the theories that give more significance to (in conditions of instability and turbulence) the social context of the universities as organizations.
\end{abstract}

Key words: Organization. University. Change. 


\section{Introducción}

En un contexto dominado por grandes transformaciones que socavan la estabilidad de las universidades, el problema que hoy preocupa a los tomadores de decisiones es descifrar: ¿cómo entender y manejar el cambio?, ¿cómo renovar su orientación, sus contenidos, su organización y la estructura que dan cuenta de su funcionamiento? La búsqueda de respuestas provoca que el tema del cambio ocupe un papel preponderante en la agenda de la investigación académica para indagar su lógica y proponer una forma para gestionarlo. En el interés de aportar nuevos elementos que abonen al conocimiento del tema en cuestión, el presente documento incluye el primer avance del proyecto de investigación: Gestión del Cambio en la Universidad Autónoma de Sinaloa (UAS), integrado por resultados del estudio exploratorio enfocado en identificar la percepción de los académicos, respecto al cambio institucional en la universidad.

La investigación se enmarca en la teoría de la organización y en los enfoques sociólogos que encauzan el entendimiento del cambio social y organizacional. Más aún, se incursionó en el plano sociotécnico analizando el instrumental de intervención que este campo del conocimiento está generando.

Este trabajo se compone de tres apartados. En el primero se conceptualiza la idea del cambio, describiendo algunas posturas que debaten sobre este fenómeno, bajo el enfoque de la teoría organizacional y estableciendo los supuestos que definen la lógica del cambio universitario. En el segundo apartado se describe de manera sucinta a la UAS como sujeto de estudio. Mientras que en el tercero se reportan los hallazgos preliminares sobre la manera en que los académicos de la UAS perciben las políticas y la forma en que asumen su compromiso con los procesos del cambio institucional.

\section{Conceptualización de cambio y la teoría organizacional}

Una de las propiedades más sobresalientes de la época actual es la velocidad y profundidad del cambio social, que conlleva a un rompimiento con el hábito y la rutina, a una obligación de pensar de manera renovada sobre temas familiares y de volverse a plantear viejos supuestos (HABERMAS, 1973, p. 10). Para analizar el significado del cambio, conviene señalar que éste se refiere a permutar una situación por otra, por lo que se le concibe como una acción que cualifica la naturaleza del ser humano y, al igual que el crecimiento y el desarrollo, es inherente a todos los individuos, grupos e instituciones; el cambio, en esta perspectiva, es la máxima expresión de la necesidad innata de alterar equilibrios establecidos, de generar nuevas ideas y distintas situaciones.

Por lo que hablar del cambio como necesidad innata de los seres humanos, no significa en absoluto que el cambio deseado pueda darse por sí mismo, ya que existe un conjunto de factores que lo determinan y lo hacen posible, los cuales emanan de los sujetos y las condiciones contextuales en que se encuentran insertos, con las cuales interactúan cotidianamente y cuyas necesidades, requerimientos y presiones producen crisis $\mathrm{y}$ conflictos, tanto en un plano interno como en uno externo, capaces de motivar el cambio.

En la teoría organizacional, la comprensión del cambio de organizaciones como la universidad se encuentra ligada al estudio de la sociedad y sus procesos; y se explica a través de tres vertientes: la sociológica, orientada a comprender el fenómeno social en todas sus manifestaciones; la administrativa y de disciplinas afines, interesadas en encontrar formas nuevas y cada vez más eficientes para que las organizaciones alcancen 
sus objetivos; y la de relaciones humanas, que nace de la preocupación por conocer y manipular los factores que inciden en su productividad, y que derivan en el reconocimiento de la importancia que reviste el comportamiento grupal de los individuos en su ambiente de trabajo.

En la idea de avanzar hacia la formulación de un modelo de intervención y gestión del cambio de la universidad, el presente trabajo encuentra en el paradigma denominado autorreferencia estructural, de Luhmann (1997, p.58) y Maturana (1990, p. 87), la plataforma adecuada para integrar las diferentes posturas teóricas y profundizar en el entendimiento de la lógica que explica el cambio de las instituciones.

En Luhmann (1982, p. 95), la teoría de los sistemas complejos y de la noción de autopoiesis, le permite explicar lo social como un sistema que aprende, que se autogenera y se autoorganiza. De forma complementaria, Morin (1993) seguido por Carrizo (2003) vendrá a precisar la idea de la complejidad como un sentido de método para establecer un soporte más equilibrado, en cuanto al entendimiento de la relación que se establece entre la organización y el contexto en los procesos de cambio. En Morin, el concepto de organización incorpora la idea organizacional de la complejidad, al asociar tres elementos: La interacción, alude a los tipos y formas de unión entre los elementos o individuos, entre estos elementos/individuos y el todo, El sistema, entendido como una unidad compleja del todo interrelacionado, que incluye caracteres y propiedades fenoménicas; la organización, como disposición de las partes dentro, en y por un todo (MORIN, 1993, p.115).

Ahora bien, para los efectos de este estudio cobra significado concebir a la universidad como un sistema, entendido como aquél que debido a la acción de las variables externas entra en movimiento, de tal modo que en su interior se generan fuerzas que inciden en su transformación, y ésta afecta también a su contexto. De lo anterior, se desprenden algunos supuestos que ayudaron a abordar los elementos relacionados con la gestión del cambio universitario.

Un primer supuesto, parte del hecho de que la universidad transita por un proceso de restructuración y diferenciación, sin haber logrado aún contar con los dispositivos de interconexión, coordinación y control adecuados para contener la desintegración y manejar su complejidad. Este supuesto incluye que las distintas formas de diferenciación o desdoblamiento de los programas de formación profesional y de investigación, en la universidad, deberán ser contenidos por modos también diferentes de integración, cuyo diseño organizacional permita armonizar y encauzar el proceso de diferenciación y especialización, evitando el riesgo del debilitamiento orgánico o del crecimiento de grandes aparatos burocráticos de coordinación y control.

En este presupuesto, la universidad estaría buscando procesar el cambio de su diseño organizacional, tomando en consideración tres elementos plenamente identificados: la diferenciación horizontal (oferta de carreras en sus diferentes modalidades), la diferenciación vertical (distintos niveles de formación y especialización), y la dispersión espacial, en términos de desconcentración y descentralización. ${ }^{\text {ii }}$

A la par de lo anterior, el proceso de cambio conducido igualmente implicaría valorar, como mecanismos de integración: la planeación y la gestión de aspectos relacionados con la estructura formal, los roles de autoridad y enlace, la comunicación, la cultura organizacional y la asignación de recursos. Esto, para evitar que la institución se transforme en un conjunto inorgánico de facultades, departamentos o unidades académicas. Un segundo supuesto se relaciona con la gestión y toma de decisiones. Tema que, desde la perspectiva de la doble contingencia observada por Luhmann, ve determinada su eficacia por la disposición de herramientas adecuadas para manejar la disyuntiva que plantean las múltiples alternativas y riesgos presentes en el devenir de las universidades, sumidas en 
condiciones de incertidumbre. Se trata de una perspectiva donde la calidad de la gestión, y la certeza de la toma de decisiones, estarán influenciadas por la observancia del patrón que acompaña a las trayectorias de cambio que han experimentado ese tipo de organizaciones.

Un tercer supuesto, hace alusión al entendimiento del poder y el liderazgo, que al tener como referente el principio de la doble contingencia, su entendimiento se verá obstaculizado por las circunstancias cambiantes en que se ve subsumida la relación del contexto y la organización, modificando los términos de actuación de la organización, por una parte, y de sus propios miembros, por la otra.

Un cuarto supuesto, busca dar soporte a un nuevo modelo de gestión del cambio de la UAS, y hace referencia a la comunicación por ser uno de los ejes más importantes que impulsan el desarrollo organizacional, al favorecer el aprendizaje de la organización y del contexto, al ayudar a fomentar el compromiso y la participación de todos los integrantes en la consecución de su misión. En tal sentido, la formulación y ejecución de un plan de comunicación e información cobra relevancia, debido a que la organización debe persuadir e informar permanentemente a sus miembros, a la vez que debe motivarlos en la ejecución de acciones encaminadas a lograr cambios.

\section{Caracterización de la Universidad Autónoma de Sinaloa.}

Actualmente a la UAS se le reconoce como una universidad pública, autónoma y descentralizada del Estado. Institución que tiene presencia en toda la entidad federativa en que se inscribe. En el ciclo escolar 2006-2007 la UAS atendió a un total de 107,556 estudiantes (licenciatura $43.03 \%$, bachillerato $37.54 \%$, posgrado $1.12 \%$, enseñanzas especiales $17.17 \%$, técnico superior universitario $0.66 \%$, y medio profesional $0.48 \%$ ).

El personal universitario de esta casa de estudios se conforma por un total de 6,279 profesores (3, 173 de tiempo completo y 3,106 de asignatura). En el mismo periodo la institución reporta 529 profesores con reconocimiento de perfil deseable y 105 investigadores miembros del Sistema Nacional de Investigadores. De la organización de la investigación se cuenta con 79 Cuerpo Académicos o grupos de investigación (CA), de los cuales 10 de ellos tiene el reconocimiento de CA consolidados.

En materia de difusión y extensión de la cultura y los servicios, la UAS ha trabajado para difundir, divulgar, promover y fomentar el aprecio por las artes, la ciencia, la tecnología y las humanidades entre la comunidad universitaria y la sociedad. Aunque de manera incipiente, son aprovechadas las nuevas tecnologías de la teleinformática y comunicaciones, se tienden a ampliar la cobertura de la vinculación con los sectores productivos, donde el servicio social que llevan a cabo los alumnos todavía mantiene un sentido de solidaridad hacia los sectores vulnerados por la pobreza y la inequidad.

En contraparte, en el plano de la gestión académica y administrativa, la UAS es observada desde el exterior como una institución de grades dimensiones, sumergida en un complejo proceso interno de desarrollo, con una comunidad altamente conflictiva y politizada, con una conducción sustentada en un modelo administrativo y académico caduco (CIEES, 1994 y 1998). Esta visión crítica del funcionamiento de esa universidad, la obliga a iniciar a partir del año 2000, un franco proceso de cambio que se implementa sin tener en ese momento una idea clara de proyecto educativo alternativo, que oriente y promueva procesos innovadores en su quehacer académico. Con respecto a las condiciones de financiamiento, los ingresos de esta casa de estudios provienen principalmente de dos fuentes: el subsidio del gobierno federal $(85 \%)$ y subsidio del gobierno estatal $(12 \%)$ y en una menor proporción de ingresos propios (3\%). 


\section{El cambio de la Universidad en la percepción de los académicos}

De acuerdo con Brunet (2003, p.12) un punto de partida para instalar una propuesta de reforma en las universidades, es el reconocimiento de los niveles de actitud y predisposición al cambio que se han generado al interior de las instituciones. Esto, como resultado de la interacción de las iniciativas institucionales de transformación y la percepción o recepción que han hecho los académicos a tales iniciativas. De acuerdo con ese autor, calibrar el grado de maduración de las iniciativas de cambio significa considerar los componentes que definen las iniciativas o proyecto institucional de transformación, y el dispositivo de gestión que lo promueve; en conocer el tipo de efectos que tienen en el sector institucional más significativo (el académico) tales iniciativas en términos de percepción, aceptación y resistencia.

Con fundamento en lo anterior, se llevó a cabo un primer acercamiento al fenómeno mediante un trabajo de exploración, a través de una encuesta. ${ }^{\text {iii }}$ El cuestionario fue diseñando tomando en consideración los distintos componentes que hoy distinguen las propuestas de modernización de la educación superior y de las universidades públicas en México, que de acuerdo con Pedroza (2004, p.18), se objetiviza en cambios curriculares y nuevas formas de ejercer la docencia, la investigación y la vinculación; en la alteración de la relación oferta-demanda educativa; en el diseño de modelos educativos innovadores; en la manera de hacer planeación y formas de financiamiento; en las formas de gobierno que implican la regulación del trabajo académico y de accesibilidad al poder.

Previo a mostrar los resultados obtenidos, es preciso anotar las consideraciones que dan fundamento al análisis de los datos que arroja la encuesta. En primer término, el estudio hace referencia principalmente a los cambios que se han presentado en la UAS en los últimos cinco años, aunque algunos se hayan dado con antelación como efectos de las tendencias generadas en las últimas décadas y que en muchos casos llevan varios años buscando su plena implementación. Una segunda consideración, tiene que ver con la naturaleza del estudio exploratorio, al buscar tener una primera aproximación a los cambios que están ocurriendo en la institución, vistos más como situación que como procesos. Ya que una evaluación profunda de dichos procesos, exige la búsqueda y análisis de nueva información, de la forma en que está operando el cambio y la manera de intervenirlo, sobre el papel que está jugando los sujetos y actores institucionales. Tareas a abordar en la segunda fase del proyecto de investigación, en correspondencia con los supuestos mencionados en el primer apartado de este artículo.

\section{Ámbito académico}

Uno de los aspectos más tangibles del cambio en esta institución está referido al currículo de las escuelas profesionales, específicamente a su plan de estudios. El $75.7 \%$ de los encuestados declaró que su plan de estudios presentó cambios en los últimos cinco años. Acepta que se actualizó considerando el acelerado desarrollo científico y tecnológico en las áreas de conocimiento que involucran la formación de los estudiantes (64.16\%); el dinamismo del mercado laboral (72\%); las aptitudes y condiciones objetivas de los estudiantes $(56 \%)$; la formación de actitudes y capacidades que permiten a los estudiantes resolver de manera eficiente y eficaz y con sustento ético las problemáticas propias de su trabajo profesional, de su vida personal y social. Así mismo, los encuestados opinaron (64.7\%) que en la actualización curricular se mejoró su pertinencia social, realizando 
estudios previos que involucran los indicadores socioeconómicos del contexto y la evaluación que presenta la profesión en cuestión.

Como puede apreciarse en la percepción de los académicos, el plan de estudios del nivel licenciatura en la UAS ha cambiado, teniendo como principal referente la visión que los actores tienen (profesores) de su contexto, el cual es recuperado en la actualización de sus planes de estudio. Aunque cabe aclarar que la actualización de estos planes de estudio obedece en cierta medida a la política que ha adoptado la institución en la búsqueda del reconocimiento externo de la calidad, a través de evaluaciones externas.

Para ser considerados de calidad, los Programas Educativos (PE) de nivel licenciatura requieren tener sus planes de estudio actualizados, y para el caso de los estudios que oferta la UAS en este nivel educativo, dicha calidad ha sido reconocida por la evaluación de organismos externos como los Comités Interinstitucionales de Educación Superior (CIEES, 2007) y por los organismos afiliados al Consejo para la Acreditación de la Educación Superior (COPAES, 2007).

Teniendo en cuenta ese reconocimiento de la calidad de los PE de licenciatura de la UAS, la institución atiende actualmente al $82 \%$ de su matrícula de nivel licenciatura en programas de calidad, tal y como lo constatan los CIEES (2007) al ubicarlos en su evaluación en el Nivel 1, o por la acreditación otorgada por COPAES (2007); ambos organismos establecen como condición previa la actualización del plan de estudios de los PE. Según datos reportados en el PIFI 2007, de un total de sesenta y tres PE de licenciatura que oferta la universidad cuarenta y siete están en el Nivel 1 de los CIEES y doce han sido acreditados por COPAES.

No obstante la importancia que revisten estos procesos de evaluación y actualización curricular, el estudio reporta que una porción significativa de los docentes involucrados en su implementación aún no participan ni se encuentran debidamente enterados de los efectos positivos que conllevan. Esto evidencia que las estrategias de comunicación y socialización de los cambios curriculares en las escuelas y facultades no son suficientes, sobre todo porque se considera que este rubro del plan de estudios les es más inmediato a los profesores, quienes dedican la mayor parte de su carga académica a ejercer la docencia. Los profesores que participaron en el cambio del plan de estudios ascienden a $74.5 \%$, porcentaje que se eleva en la actualización de programas de su materia (83.6\%) y en los ejercicios de autoevaluación interna $78.5 \%$; estos porcentajes si bien es cierto son altos están todavía muy lejos del ideal buscado; esto es, involucrar a todos los profesores en la revisión y actualización de sus planes de estudios.

En lo que respecta a la investigación, una baja proporción de los encuestados (40.1\%) reconoció que accede a los beneficios de los programas de apoyo e incentivos para los profesores implementados por esta universidad; tal es el caso del Programa de Mejoramiento del Profesorado (PROMEP) y del Programa de Estímulos al Desempeño Académico. La formación y consolidación de los Cuerpos Académicos (CA) es otro de los cambios valorados en este rubro, donde apenas el $47 \%$ declaró ser miembro de un CA; este escaso porcentaje de participación está en consonancia con los datos considerados en el Programa Integral de Fortalecimiento Institucional (PIFI) 2007, mas su inconsistencia se reconoce cuando de los ochenta y siete CA registrados por la UAS ante PROMEP sólo seis se han consolidado. No obstante, el $70 \%$ de los integrantes de CA manifestó que participa en un proyecto de investigación orientado a la generación o aplicación de conocimientos científicos y/o humanísticos, lo que tendría que verse con reservas debido al escaso desarrollo presentando por la investigación en la Universidad Autónoma de Sinaloa.

La oferta y demanda educativa es otro de los rubros considerados en la encuesta; se explora la opinión de los docentes en torno a los procedimientos de ingreso y selección de 
estudiantes, donde los mecanismos de ingreso diseñados por las escuelas alcanzaron el $60 \%$ de opiniones favorables, mientras que $71.5 \%$ consideró que es adecuada la aplicación del examen de CENEVAL, ya que elevan la calidad de la educación que se imparte (56.9\%) y mejoran la orientación vocacional de los estudiantes (61.6 por ciento).

En tanto, el $72.4 \%$ opinó que las políticas de ingreso a los PE que oferta la UAS garantizan la igualdad de oportunidades a estudiantes, sin distinción de su condición social. Aunado al diseño e implementación del programa de tutorías, becas económicas, servicios asistenciales, cursos remediales, servicios médicos, entre otros apoyos que brinda la institución a los estudiantes para que logren los objetivos académicos a lo largo de su trayectoria escolar y permanezcan en la institución. Aunque, también, los encuestados se manifestaron a favor de la ampliación de la cobertura del servicio educativo de nivel superior $(69.9 \%)$, a través de la implementación de modalidades alternativas a la presencial, como es el caso de educación abierta o semipresencial.

Esto da cuenta de un conocimiento de la presión de la demanda educativa que tiene la UAS para incrementar su matrícula estudiantil en el nivel superior, y el reconocimiento de que la institución, con la organización académica y administrativa que hoy tiene, no puede responder adecuadamente a tales requerimientos, lo que lleva a explorar nuevos proyectos educativos de modalidad abiertos y no escolarizados. Aprovechando las ventajas que brinda la telemática, la universidad puede convertirse en bimodal, esto es, potenciar sus campus virtuales para apoyar ambas modalidades, al igual que los sistemas de información y comunicación con finalidades formativas deberán ser flexibles, tomando en cuenta el tiempo y espacio, interactivos y personalizados. Aquí, cobra importancia la capacitación permanente de los egresados y de profesionistas a través de la educación continua, aunque apenas alcanzó un $54.9 \%$ de reconocimiento por parte de los profesores encuestados.

El modelo pedagógico es otro rubro considerado en la evaluación de la dimensión académica en la UAS que integra el cuestionario; llama la atención los bajos resultados registrados, de donde se infiere que la oferta educativa de nivel licenciatura en la UAS sigue operando con un modelo pedagógico obsoleto y tradicional, ya que los rasgos que definen un modelo alternativo centrado en el aprendizaje de los alumnos, fueron desagregados y presentados para efectos de valoración de los encuestados, cuyos resultados se muestram a continuación. La percepción de los académicos que ilustran lo anterior, se refiere a las respuestas vertidas para conocer si el modelo pedagógico con el que se implementa la oferta educativa de nivel superior en la UAS pone en el centro del proceso educativo al estudiante, promoviendo su formación integral que permite el desarrollo armónico de sus esferas intelectual, física, ética, afectiva y estética; el $53.3 \%$ opinó favorablemente; sí fomenta aprendizajes significativos que los preparan para la vida, alcanzó apenas el 54\%; y sí genera competencias profesionales fincadas en actitudes y valores para atender la problemática social, el trabajo en equipo y el respeto a la diversidad registró un 59\%. Por último, en la operacionalidad del modelo educativo, se indagó sobre la participación del estudiante en la determinación de su ruta académica, estando de acuerdo con ello sólo el 52.2\% de los docentes considerados en el estudio.

Debido a ello, consideramos que estos aspectos relacionados con la implementación de un modelo educativo alternativo, centrado en la enseñanza que asegura la formación integral de los estudiantes, es un área que la UAS requiere seguir trabajando, aunado al diseño curricular por competencias, a la búsqueda de su apertura y flexibilidad curricular, aspectos que siguen siendo una asignatura pendiente que habrá de reforzar en la estrategia del cambio en la institución. 


\section{Ámbito de la gestión y administración}

En lo que a planeación institucional concierne, y en tanto que es considerada un vehículo para la clarificación de la misión y la visión de las instituciones, para aumentar la capacidad para enfrentar desafíos y promover cambios a través de la elaboración, seguimiento y evaluación de los planes estratégicos de desarrollo, se valoró primeramente en este rubro si la actual Administración Central posee una visión estratégica de futuro (59.9\%), si la planeación institucional ha contribuido al diseño y promoción de propuestas y cambios significativos en la UAS (66.4\%); si los planes elaborados integran las necesidades presentes y futuro del desarrollo regional (60.8); y si éstos han contribuido en la modernización e innovación de los procesos académicos y administrativos de la universidad $(60.06 \%)$. Por otra parte, se preguntó además si en los procesos de planeación (elaboración, implementación, seguimiento y evaluación de los planes de desarrollo) se han creado los mecanismos y las estancias adecuadas que garantizan la participación de la comunidad universitaria (50 por ciento).

No obstante que en la UAS la elaboración, seguimiento y evaluación del Plan Institucional y de los Planes Estratégicos de Unidades Organizacionales se implementan con un Modelo de Planeación Estratégica y de Dirección por Objetivos, y que esta universidad lo ha asumido como un proceso, el cual ha sido documentado y certificado en el 2003 bajo la familia de Normas ISO 9001:2000, en el marco de la implementación de su Sistema de Gestión de Calidad, y que se tienen productos importantes en este renglón, desde la opinión de los encuestados no se aprecian aún, suficientemente, los beneficios que se derivan de la aplicación de ese enfoque de planeación, como son fomentar el pensamiento y la acción estratégica de los directivos e integrantes del colectivo de una organización; mejorar el proceso de toma de decisiones, apoyar el proceso de formulación y comunicación de la estrategia de desarrollo; mejorar la capacidad de desempeño y las respuestas de la organización frente a las presiones generadas en su interior y por demandas externas emanadas de un contexto que cambia profundamente a un ritmo acelerado.

En términos generales la planeación institucional, de acuerdo con las respuestas emitidas por los encuestados, no se ha convertido aún en un instrumento propio que oriente desde una posición interna el quehacer académico y administrativo de la institución, y que fundamente la toma de decisiones de su alta dirección; tampoco se le ve como un sistema integrado ni como entidad que reúne el carácter participativo e integral, ya que el $70.7 \%$ de los profesores considerados en la muestra manifestaron que la conducción institucional de la UAS continúa rigiéndose por los lineamientos que emanan de la política de la Secretaría de Educación Pública - SEP- (2001) hacia este sector educativo.

Esto lleva a ubicar la planeación de la reforma institucional como un área crítica; por ello, es deseable el reforzamiento de los requerimientos que aseguren en la institución una planificación exitosa, que involucran el liderazgo en todos sus niveles de mando, la participación colectiva de su comunidad universitaria; el buen funcionamiento de sus órganos de gobierno, la corresponsabilidad en el funcionamiento de unidades académicas y administrativas; el acopio de capital humano interno en este campo del conocimiento; y, también, el apoyo social externo.

En lo que se refiere a legislación institucional, la opinión expresada de parte de los encuestados es el reconocimiento (65.9\%) de que la UAS cuenta con un cuerpo normativo que define con suficiencia su existencia, carácter (público, autónomo) y personalidad para cumplir a cabalidad con sus fines, así como para regular su conducción y funcionamiento. 
Para ahondar en el reconocimiento de la legislación universitaria, el estudio indagó sobre si el marco normativo ha experimentado en los últimos años cambiosiv que contribuyen a la mejora del trabajo académico, la eficiencia administrativa y la apertura de la universidad a la sociedad $(57.70 \%)$, a fortalecer formas colegiadas de coordinación del trabajo académico $(62.0 \%)$; a promover la descentralización de la estructura académica y administrativa y la distribución de la autoridad y el ejercicio del poder (44.70), a garantizar una elección y designación de las autoridades universitarias en un marco de participación democrática y de estabilidad institucional $(48.70 \%)$. También se preguntó si en la actual administración se avanza en la elaboración de normas que mejoren la regulación del ingreso, promoción, capacitación, aprovechamiento y desempeño del personal académico, administrativo y directivo (51.30 por ciento).

Cabe destacar que, aunque se sabe que es deseable que el marco normativo de la institución sea adecuado y suficiente para garantizar el desarrollo orgánico de la institución y para que ésta pueda dirimir los conflictos, tanto internos como externos, que cotidianamente se le presenten, contrastan las respuestas que al respecto emitieron los encuestados, registrando porcentajes menores en relación a los porcentajes más altos del estudio.

Aunque la interpretación de la baja opinión favorable de los profesores encuestados sobre el sentido de las reformas normativas puede ser diversa, en ello se vislumbra la hipótesis de que en la UAS la estructura y estilo de gestión históricamente establecidos no ha generado la calidad de las instancias y los espacios adecuados para decidir con sentido de comunidad sobre los grandes problemas del desarrollo universitario. En tal sentido se inscribe la opinión de los profesores encuestados sobre la falta de participación del colectivo universitario sobre el tema referido, y sobre la percepción que ellos tienen de su socialización y difusión en la comunidad universitaria (56.70 por ciento).

En contraparte, la normatividad referida a los estudiantes resultó mejor valorada, ya que el $72.80 \%$ opinaron que en la UAS se cuenta con políticas y criterios claros y normados en materia de ingreso, permanencia, egreso y opciones de titulación de sus estudiantes.

A partir de este nuevo marco normativo la institución inicia, aunque con lentitud, un proceso de reorganización formal de su estructura académico-administrativa, con el propósito de optimizar el uso de recursos (humanos, materiales y financieros) y favorecer la desconcentración, descentralización y regionalización de su actividad académica. Por ello, en el análisis de su dimensión de gestión y administración, este punto cobra renovada importancia en el acopio y análisis de la información derivada de la encuesta, mostrando los siguientes resultados.

Los encuestados no tienen una noción clara del modelo educativo propuesto para la institución por la actual administración, el cual es la columna vertebral del Plan Buelna de Desarrollo Institucional 2005-2009: Propuesta para un nuevo modelo educativo centrado en la calidad. A juzgar por los bajos porcentajes alcanzados en la encuesta, cuando se cuestionó si en la actual administración el cambio de la universidad ha estado orientado por un nuevo modelo de organización académica, curricular y pedagógica, que da pauta a la innovación, la diversificación y el fortalecimiento de la calidad de la formación profesional $(62.1 \%)$; si se cuenta con una metodología adecuada para poner en consonancia los planes de estudios a los requerimientos que plantea el nuevo paradigma educativo; si atiende al dinamismo del contexto y los fines de la institución (51.30\%). Para ello, se interrogó si la UAS cuenta con políticas y las instancias adecuadas para apoyar la evaluación y la actualización de los planes de estudios y mejorar la coordinación del trabajo académico (57.30 por ciento). 
Por otro lado, también se preguntó si como parte de la reforma curricular y pedagógica que se opera en la institución se cuenta con un programa permanente de formación y actualización de profesores $(51.40 \%)$. Llama aquí la atención el bajo porcentaje de académicos que percibe la presencia de programas de formación de sus académicos, toda vez que se reconoce que para operar un nuevo modelo pedagógico centrado en el estudiante requiere de nuevas estructuras académicas y administrativas, del aprovechamiento de las nuevas tecnologías, y sobre todo de la actualización y formación de los docentes.

Debido a que la gestión de los nuevos entornos de aprendizaje y el cambio metodológico de la enseñanza exigen un cambio de mentalidad y de prácticas docentes, que involucra métodos alternativos de enseñanza que restituyen el papel activo del estudiante en su propio aprendizaje, lo cual a menudo superan los muros del aula y de la escuela; aprovechan las posibilidades de las nuevas tecnologías (TIC); proponen el desarrollo de proyectos colaborativos, apoyándose en la utilización de materiales de multimedia y fomentando sistemas de autoaprendizaje. Con el uso de las TIC se liberan horas de clase y se pueden dedicar más tiempo a otro tipo de actividades académicas, por ejemplo, la atención más personalizada a los alumnos a través de la tutoría y de asesorías grupales.

En esta perspectiva de formación, los roles asignados a los docentes, son: diseñador y gestor de actividades y entornos de aprendizaje; orientador, guía de aprendizajes y del desarrollo de las capacidades de los alumnos; motivador, provocador de curiosidad intelectual y entusiasmo, dinamizador de los grupos; fuente de información y de conocimientos; transmisor de experiencias; creador de recursos pedagógicos (diseño y desarrollo) y motivar su uso; co-aprendiz con los estudiantes, promoviendo descubrimientos guiados; tutor; investigador que reflexiona sobre su práctica y colabora con otros docentes; participa en la revisión y actualización de los contenidos de su asignatura, y de los planes de estudios. Así, al profesor universitario se le emplaza a ejercer una práctica docente diversificada que comprende docencia, investigación y gestión.

Salta a la vista que este nuevo rol de los docentes tiene que ir acorde con nuevas condiciones de trabajo, por lo que se consideró oportuno conocer si la UAS cuenta con un programa adecuado de actualización y mantenimiento permanente de la infraestructura académica. $(50.40 \%)$; y si la institución posee e implementa un programa (modernización tecnológica) de información y comunicación institucional orientado a fortalecer la generación, transmisión y difusión del conocimiento y las relaciones de intercambio académico institucional, local, nacional e internacional (55.20 por ciento).

En el mejoramiento de los procesos académicos y administrativos, los encuestados opinaron que la UAS también se rige por los dictados de la SEP, lo que implica limitar los cambios a la eficientización de lo tradicional (63.80\%). De ahí la pertinencia de emplazar a los profesores considerados en este estudio para que manifiesten su opinión en torno a los principales ejes estratégicos (acreditación de programas educativos y certificación de procesos administrativos) que articulan la propuesta de desarrollo de la actual administración universitaria, incluyendo al ámbito académico y administrativo.

Concretamente, se indagó si la política de acreditación de programas educativos que ha emprendido la UAS ha mejorado su calidad académica (68.50\%), y si la certificación de procesos administrativos que ha llevado a cabo en los últimos dos periodos rectorales ha mejorado la calidad del servicio que se presta a los estudiantes (49.80\%). Nótense los bajos porcentajes registrados en ambos aspectos, mismos que hacen pensar que, desde la opinión de los encuestados, la acreditación de los programas educativos y la certificación de procesos administrativos no han impactado lo suficiente para cambiar los 
principales actividades que llevan a cabo los actores universitarios, para dar cumplimiento a las funciones sustantivas y de apoyo de la universidad.

Otro aspecto que indaga la encuesta es el financiamiento, su diversificación, uso y manejo, donde los encuestados a juzgar por sus respuestas cuestionaron este rubro en la universidad, en tanto que se les preguntó si la UAS aprovecha suficientemente las oportunidades de financiamiento alterno $(38.40 \%)$; si existe un control financiero, con mecanismos de fiscalización y de control (interno y externo) que aseguran su buen uso y transparencia $(29.80 \%)$; si la gestión financiera se rige por políticas y prioridades para la asignación del gasto, con base en el presupuesto anual por programa, sancionado por el $\mathrm{H}$. Consejo Universitario (37.50\%); si la conducción institucional logra sensibilizar y hacer partícipes a los sectores universitarios, padres de familia y egresados en el mejor aprovechamiento de los recursos y en una mayor contribución con la institución, a través de colegiaturas, donaciones y aportaciones diversas (36.6\%).

Aunque se sabe que en este renglón la universidad ha emprendido acciones para diversificar sus fuentes de financiamiento y trasparentar el buen uso de los recursos, como son la certificación de los procesos del área financiera de su Administración Central, la existencia de la Controlaría Social, la realización de auditorías financieras y académicas tanto internas como externas; no obstante, éstas continúan sin tener efecto en la percepción positiva de los universitarios considerados en el estudio.

La gestión que la institución ha realizado con los sectores productivos, sociales y académicos, fue mejor vista por los encuestados, quienes manifiestan que la UAS ha incrementado las relaciones de intercambio, cooperación y de coordinación con otras IES, lo cual incluye programas de movilidades de estudiantes y profesores $(72.90 \%)$; asimismo valoran la ampliación del marco de relación y diálogo establecidos con otros sectores sociales que atienden su sentir en la definición de la política de desarrollo académico apoyándola en su concreción (62.50\%); aprecian cómo se incrementa la participación de académicos en convenios y contratos de intercambio y vinculación: prácticas y/o servicios profesionales, estancias, educación continua, investigación tecnológica (65.50\%).

Por último, se incluyen en el cuestionario aspectos relacionados con la función de gobierno de la institución, indagando en la encuesta lo siguiente: si en la actual administración se ha mejorado la comunicación de la Administración Central y directores de escuelas con profesores, estudiantes, trabajadores egresados y sectores de la sociedad $(51.50 \%)$; si se promueve más la participación y es mayor el interés de la comunidad universitaria por participar en la toma de decisiones (41\%); si las autoridades universitarias cuentan con liderazgo y capacidad requerida para transmitir su política de desarrollo e identificar a la comunidad universitaria con la misión de la UAS y el proyecto de nueva universidad que se busca construir (47.80\%). Si la actual administración de la UAS practica una gestión transparente, participativa y de rendición de cuentas a la sociedad $(40.60 \%)$. 


\section{Conclusiones}

Desde la visión sistémica que se asume en esta investigación, se exploró la percepción que tienen los académicos del cambio que está experimentando la Universidad Autónoma de Sinaloa, a través de la valoración del comportamiento que presenta el conjunto de sus componentes, para comprender sus transformaciones como un todo buscando esclarecer la dinámica de su interrelación e interdependencia, ya que cuando los componentes del sistema interactúan adecuadamente se alcanzan mejores niveles de desarrollo, y para el caso de la UAS dicho desarrollo ha sido demostrado con la elevación de los índices de los indicadores de desempeño relativos a su competitividad y capacidad académica.

A partir de lo cual, se infieren las siguientes conclusiones: primera, la dimensión académica conforma el ámbito del cambio mejor percibido por sus docentes; segunda, se valoran como positivas las nuevas formas de ejercer la docencia, la investigación y la vinculación; tercera, se presenta un interés aceptable de los académicos en adoptar un nuevo modelo educativo en la institución; cuarta, la política emitida por la SEP para transformar la educación superior en el país, conforma el principal referente para orientar el cambio en las escuelas que integran a la UAS.

En contraste con lo anterior, la quinta conclusión nos muestra que existe un menor nivel de aceptación de los cambios que involucran el desempeño de la dimensión de administración y gestión; mientras una sexta advierte un bajo reconocimiento de los académicos a los mecanismos de integración institucional, como son la normatividad, estructura de poder y coordinación (centralismo), liderazgo, la planeación y el proyecto de universidad, toma de decisiones, mecanismos de comunicación, confianza y transparencia en la gestión, además de asignación y uso de recursos.

Lo anterior sirve de fundamento para refrendar la hipótesis con la que se dará continuidad a este proyecto de investigación, de que en la UAS el proceso de cambio conducido, al tener como principal referente los programas y políticas que la SEP emite para este nivel de educación superior, está debilitando los mecanismos de integración. Por lo que la dinámica de cambio que se viene produciendo en el ámbito académico, al no tener como fuente de su gestión un proyecto propio de nueva universidad, que estructure y encause con sentido de comunidad el quehacer académico, está debilitando los factores institucionales de identidad, coordinación, integración y generación de espacios de cooperación entre las Unidades Académicas que conforman esta institución de educación superior.

En síntesis, en la UAS los procesos de cambio son motivados principalmente desde el exterior, siendo generadores de fuerzas de cambio que carecen de un plan estratégico efectivo y de un modelo de gestión que encause, promueva y controle el desempeño institucional. Aunque vale pena relativizar el peso asignado a las fuerzas externas que están motivando el cambio, dado que la universidad es considerada un sistema entendido como aquél que debido a la acción de las variables externas entra en movimiento, de tal modo que en su interior se generan también fuerzas que inciden en su transformación, y el funcionamiento de ésta afecta positivamente a su contexto, la manera en que esto está ocurriendo en la UAS se convierte en el foco de las siguientes fases de esta investigación. 


\section{Referências}

BRUNET, I. La universidad y la gestión del cambio. México: Universidad Michoacana de San Nicolás de Hidalgo, 2003.

CARRIZO, L. El investigador y la actitud transdisciplinaria: condiciones, implicancias, limitaciones. En Documento de la UNESCO: Gestión de las transformaciones sociales, México: UNESCO, 2003.

HABERMAS, A. M. Cómo se realizan los cambios en la educación: una contribución al estudio de la innovación. Chile: UNESCO-OIE, 1973.

MATURANA, H. Emociones y lenguaje en educación y política. Chile: Editorial Hachette, 1990.

MORIN, E. El Método I: La naturaleza de la naturaleza. Tomo I, Madrid: Editorial Cátedra, 1993.

PEDROZA, F. R. La flexibilidad académica en la universidad pública. Revista: Confluencia, núm. 119. México: ANUIES, 2004.

UNIVERSIDAD AUTÓNOMA DE SINALOA. Dictámenes de evaluación de los CIEES de la administración y gestión institucional de la UAS. México: UAS, 1994 y 1998.

UNIVERSIDAD AUTÓNOMA DE SINALOA. Plan Buelna de Desarrollo Institucional: 2005 -2009, México: Editorial UAS, 2005.

UNIVERSIDAD AUTÓNOMA DE SINALOA. Programa Integral de Fortalecimiento Institucional-UAS. México: UAS, 2007.

SECRETARIA DE EDUCACIÓN PÚBLICA. Programa Integral para el Fortalecimiento Institucional. Lineamientos. México: SEP, 2001.

Direcciones electrónicas:

www.copaes.org.mx (Consultado en febrero 2007)

www.ciees.edu.mx (Consultado en febrero 2007)

\footnotetext{
${ }^{\text {i }}$ El término compromiso alude a los diferentes aspectos relacionados con el nivel de involucramiento y/o participación de los docentes con el proceso del cambio universitario, se integra con tres componentes: Identificación: aceptación de la dirección del cambio; Membrecía: deseo de participar en el cambio. (ser parte de); y Lealtad: cumplimiento y respeto con el cambio planeado, mediante la realización de acciones dirigidas a impulsarlo y defenderlo.

ii En este caso, descentralización significa la transferencia de autoridad y capacidad de decisión de los órganos centrales a las unidades regionales. Mientras que la desconcentración alude a los procesos de planeación, a la delegación de responsabilidades y compromisos del ejercicio de planeación. Esto implica la transferencia de recursos y apoyos administrativos.
} 


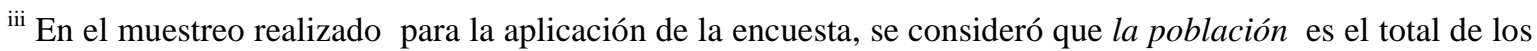
profesores universitarios del nivel profesional de las cuatro zonas en que se desagrega la UAS. Las unidades de muestreo son las escuelas y facultades, mientras que la unidad de observación son los profesores encuestados. El tamaño de la muestra fue de 205, distribuidos por zonas se aplicaron en la Centro 137, en la Centro Norte 11, en la Norte 24 y en la Sur 35. Los tiempos de aplicación de la encuesta comprenden los meses de mayo y junio de 2007.

iv En el 2006 se aprobó la nueva Ley Orgánica, la reglamentación que de ella se deriva se aprobó durante el 2007 (Estatuto General, Reglamento de Autoridades Universitarias Colegiadas, Reglamento de la Defensoría de los Derechos Universitarios, Reglamento de Funcionamiento del Consejo Consultivo y de Vinculación Social, Reglamento de Servicio Social, Reglamento de Transparencia y de Acceso a la Información, Reglamento Escolar, Reglamento de Funcionamiento del Tribunal Universitario, y Reglamento del Personal Académico).

Artigo recebido em: $22 / 01 / 2010$

Aprovado para publicação em: 10/03/2010 